\title{
Quantitative cardiovascular magnetic resonance in pregnant women: cross-sectional analysis of physiological parameters throughout pregnancy and the impact of the supine position
}

Alexia Rossi ${ }^{1,2}$, Jerome Cornette ${ }^{3}$, Mark R Johnson ${ }^{4}$, Yusuf Karamermer ${ }^{1,2}$, Tirza Springeling ${ }^{1,2}$, Petra Opic ${ }^{1}$, Adriaan Moelker ${ }^{2}$, Gabriel P Krestin ${ }^{2}$, Eric Steegers ${ }^{3}$, Jolien Roos-Hesselink ${ }^{1}$ and Robert-Jan M van Geuns ${ }^{1,2^{*}}$

\begin{abstract}
Background: There are physiological reasons for the effects of positioning on hemodynamic variables and cardiac dimensions related to altered intra-abdominal and intra-thoracic pressures. This problem is especially evident in pregnant women due to the additional aorto-caval compression by the enlarged uterus. The purpose of this study was to investigate the effect of postural changes on cardiac dimensions and function during mid and late pregnancy using cardiovascular magnetic resonance (CMR).

Methods: Healthy non-pregnant women, pregnant women at $20^{\text {th }}$ week of gestation and at $32^{\text {nd }}$ week of gestation without history of cardiac disease were recruited to the study and underwent CMR in supine and left lateral positions. Cardiac hemodynamic parameters and dimensions were measured and compared between both positions.

Results: Five non-pregnant women, 6 healthy pregnant women at mid pregnancy and 8 healthy pregnant women at late pregnancy were enrolled in the study. In the group of non-pregnant women left ventricular (LV) cardiac output (CO) significantly decreased by $9 \%(\mathrm{p}=0.043)$ and right ventricular (RV) end-diastolic volume (EDV) significantly increased by $5 \%(p=0.043)$ from the supine to the left lateral position. During mid pregnancy LV ejection fraction (EF), stroke volume (SV), left atrium lateral diameter and left atrial supero-inferior diameter increased significantly from the supine position to the left lateral position: 8\%, 27\%,5\% and $11 \%$, respectively ( $p<$ 0.05). RV EDV, SV and right atrium supero-inferior diameter significantly increased from the supine to the left lateral position: 25\%, 31\% and 13\% ( $<<0.05$ ), respectively. During late pregnancy a significant increment of LV EF, EDV, SV and CO was observed in the left lateral position: 11\%, 21\%, 35\% and 24\% ( $<<0.05)$, respectively. Left atrial diameters were significantly larger in the left lateral position compared to the supine position $(p<0.05)$. RV CO was significantly increased in the left lateral position compared to the supine position $(p<0.05)$.

Conclusions: During pregnancy positional changes affect significantly cardiac hemodynamic parameters and dimensions. Pregnant women who need serial studies by CMR should be imaged in a consistent position. From as early as 20 weeks the left lateral position should be preferred on the supine position because it positively affects venous return, SV and CO.
\end{abstract}

\footnotetext{
* Correspondence: r.vangeuns@erasmusmc.nl

'Department of Cardiology, Erasmus University Medical Center, 's

Gravendijkwal 230, 3015CE Rotterdam, The Netherlands

Full list of author information is available at the end of the article
} 


\section{Background}

Increasing numbers of women with pre-existing heart disease are reaching childbearing age and are deciding to become pregnant [1]. Pregnancy induces marked physiological changes in cardiac parameters, with a $30-50 \%$ increase in cardiac output, through an increase both in stroke volume and heart rate [2]. While usually well tolerated in healthy pregnant women, these changes can induce adverse effect in women with pre-existing heart disease on both right and left-sided lesions $[3,4]$. Therefore, heart function should be closely monitored during pregnancy in these patients. Echocardiography has been used for many years but cardiovascular magnetic resonance (CMR) is more reliable in the context of congenital heart disease [5]. To date, most data have been derived using echocardiography with the patients in lateral position [6], while CMR is usually performed in supine position. As aortocaval compression is important in advanced pregnancy $[7,8]$ data of both techniques can not be compared. Many women with complex cardiac conditions will require CMR during pregnancy, however there is relatively little data regarding both the use of CMR during pregnancy and of the impact of supine and lateral positions on cardiac parameters. The purpose of this study was to investigate the impact of maternal position on cardiac parameters derived from CMR during $2^{\text {nd }}$ and $3^{\text {rd }}$ trimesters of pregnancy in normal women.

\section{Methods}

\section{Patient selection}

Healthy non-pregnant women, pregnant women at $20^{\text {th }}$ week of gestation and at $32^{\text {nd }}$ week of gestation with no history of cardiac disease were recruited to the study between June 2009 and January 2010. Study participants underwent CMR in supine and left lateral positions. Exclusion criteria were the common contraindications for CMR studies (pacemaker, cochlea implants and claustrophobia). The study was approved by the institutional review board and each subject gave informed consent.

\section{CMR protocol}

CMR was performed using a $1.5 \mathrm{~T}$ scanner (Signa CV/I, GE Medical System, Milwaukee, WI). Firstly the patient was placed in the supine position and entered feet first into the magnet. A dedicated cardiac 8 channels coil was placed on the thorax of the subject and used for the acquisition of the images. CMR cines were obtained using a breath-holding ECG triggered balanced steady state free precession sequence. Imaging parameters were as follows: FOV $36-40 \times 28-32 \mathrm{~cm}$; matrix $224 \times 196$; TR: 3.4 milliseconds; TE: 1.5 milliseconds; flip angle 45 degrees; 12 views per segment. Slice thickness was 8 $\mathrm{mm}$ with a gap of $2 \mathrm{~mm}$. These parameters resulted in a temporal resolution per image of 41 milliseconds. At first, three rapid surveys were obtained for the determination of the cardiac position and orientation; two- and four-chamber cine MR images were then obtained. The series of short axis (SA) images were obtained from the reference images provided by the two- and four- chamber end-diastolic images at the end of expiration. Approximately 10 to 12 slices were acquired to cover the entire length of the heart. Directly after the first CMR study, the subject was repositioned on the left lateral side position for the second examination. The acquisition of images was performed by the same operator.

\section{Image analysis}

All the studies were analysed on a remote workstation using the CAAS-MRV (version 3.2; Pie Medical Imaging, Maastricht, The Netherlands). Left end-diastolic volume (EDV) and end-systolic volume (ESV) were calculated using a combination of classic SA and long-axis images. The long-axis view was used to limit the extend of volumes at the base and at the apex of the heart. The Simpson rule was used to calculate volumes based on the SA images where the first basal and the last apical were only partially included relating to the area outlined on two- and four- chamber images. More details about this approach have been previously reported [9]. The papillary muscles were considered as being part of the blood pool shortening the analysis time without compromising the accuracy of LV volumes compared to standard short-axis technique [10]. Ejection fraction (EF) was calculated as (EDV - ESV)/EDV. Cardiac output $(\mathrm{CO})$ was calculated from stroke volume (SV) and heart rate (HR). Left atrium volume (LAvol) was measured using a combination of the two- and four chamber views in the diastolic phase of the atria. Lateral (LAlat) and supero-inferior (LAsi) left atrial diameters were measured on a four-chamber view during the phase of cardiac cycle with the largest left atrium (Figure 1). The lateral diameter was taken from the perpendicular constructed from the midpoint of the LAsi diameter extending to the atrial borders. The LAsi dimension corresponds to a line bisecting the left atrium and extending from the midpoint of the mitral annulus to the midpoint of the superior left atrium.

Right EF, EDV, ESV, SV and CO were calculated using cine images acquired in SA view, parallel to the tricuspid valve annulus and applying the same methods of the left ventricular hemodynamic measurements without long-axis corrections. In addition, lateral (RAlat) and supero-inferior (RAsi) right atrium diameters were taken during the phase of the cardiac cycle with the largest right atrium, on a four-chamber view (Figure 1). The 


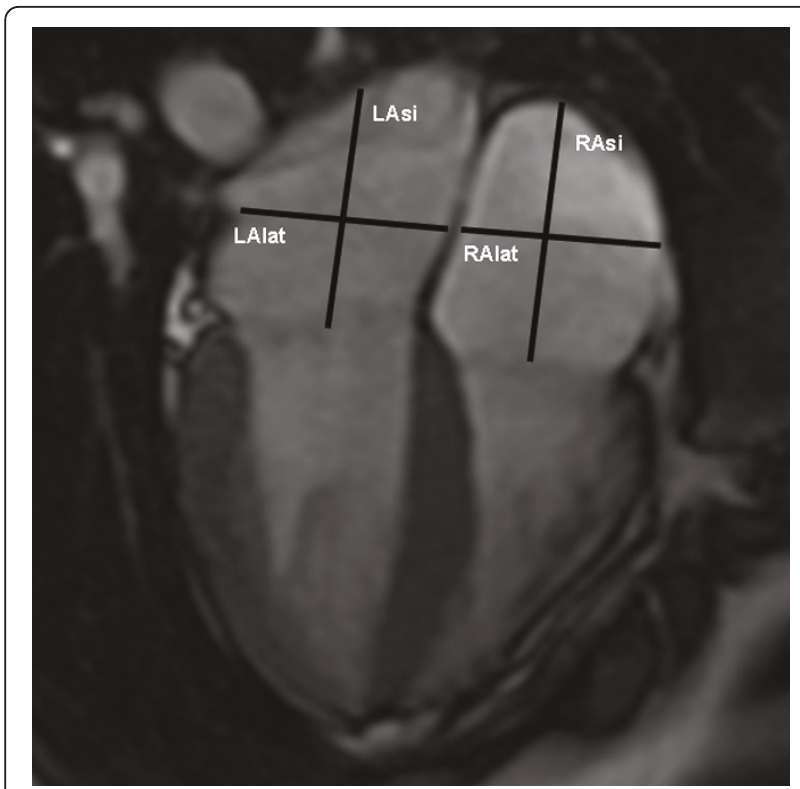

Figure 1 Cardiac dimensions: four-chamber end-systolic view LAlat: left atrium lateral diameter; LAsi: left atrium supero-inferior diameter; RAlat: right atrium lateral diameter: RAsi: right atrium supero-inferior diameter.

RAlat diameter corresponds to the line extending from the atrial borders and perpendicular to the RAsi diameter. The RAsi diameter is the line from the midpoint of the tricuspid valve to the midpoint of the superior right atrium.

Due to the low inter-observer variability reported in a previous study [11] image analysis was performed by one operator with 3 years-experience in CMR. The operator was blinded to the same patient at the other position. CMR analyses were performed in a random order at different days.

\section{Analysis by gestational week}

The patients were categorized into 3 groups according to gestational age. The first group consists of non-pregnant controls, the second group of women in the $20^{\text {th }}$ gestational week and the third group of women in the $32^{\text {nd }}$ gestational week.

\section{Statistical analysis}

All analyses were done using SPSS 15 (SPSS Inc.) software. Parametric data were reported as mean \pm standard deviation. For each gestational group mean values of HR, EDV, ESV, EF, SV, CO, left and right atrium diameters of supine and left lateral positions were tested for significance, using Wilcoxon's two sample test. A pvalue $<0.05$ was considered significant. The percentage of change in the measure of left ventricle (LV) and right ventricle (RV) parameters (X) and left atrium (LA) and right atrium (RA) parameters $(\mathrm{X})$ between supine and left lateral position was calculated using the following formula:

percentage change in $\mathrm{X}=\left[\left(\mathrm{X}_{\text {lateral }}-\mathrm{X}_{\text {supine }}\right) / \mathrm{X}_{\text {supine }}\right] \times 100$.

\section{Results}

A total of 14 healthy women with singleton pregnancies (30.3 \pm 5.2 years) were included in the study. Five nonpregnant women $(29.4 \pm 5.7$ years $)$ were recruited as controls. The time interval between the examinations in supine and left lateral position ranged between 8 and 12 minutes. Table 1 gives the data regarding hemodynamic parameters and cardiac dimensions as related to gestational week and maternal posture. Percentage differences of cardiac volumes between supine and left lateral position are graphically reported in Figure 2 and 3 and will be reported in more detail below.

\section{Pre pregnancy}

HR did show a slight although not significantly decrease between supine and left lateral position: $78 \pm 12$ versus $73 \pm 12$. Left CO significantly decreased by $9 \%(\mathrm{p}=$ 0.043 ) and right EDV significantly increased by $5 \%$ ( $\mathrm{p}=$ 0.043). There were no other significant changes of hemodynamic parameters and cardiac dimensions between the two recumbent positions.

\section{0 gestational weeks}

Six pregnant women were in the $20^{\text {th }}$ gestational week. $\mathrm{HR}$ was $80 \pm 11 \mathrm{bpm}$ in the supine position and $72 \pm 5$ in the left lateral position $(\mathrm{p}=0.15)$. A significant increment of EF and SV of the left ventricle was observed between the supine and the left lateral position: $8 \%$ ( $\mathrm{p}=$ $0.046)$ and $27 \%(p=0.028)$, respectively. Left atrial dimensions increased significantly between the supine and the left lateral position by $5 \%$ for LAlat $(\mathrm{p}=0.028$ ) and by $11 \%$ for LAsi ( $\mathrm{p}=0.027$ ). Regarding the right side of the heart, EDV increased by $25 \%(0.028)$ and SV increased by $31 \%(0.028)$ between the supine and the left lateral position. RAsi significantly increased by $13 \%$ $(\mathrm{p}=0.042)$ between the supine and the left lateral position.

\section{2 gestational weeks}

Eight pregnant women were in the $32^{\text {nd }}$ gestational week. HR did not significantly change between the supine and the left lateral position: $81 \pm 16$ versus $75 \pm$ $8 \mathrm{bpm}(\mathrm{p}=0.237)$. A significant increment of EF, EDV, $\mathrm{SV}$ and CO was observed between the supine and the left lateral position: $11 \%(\mathrm{p}=0.012), 21 \%(\mathrm{p}=0.012)$, $35 \%(p=0.012)$ and $24 \%(p=0.012)$, respectively. Left 
Table 1 Influence of position related to gestational weeks

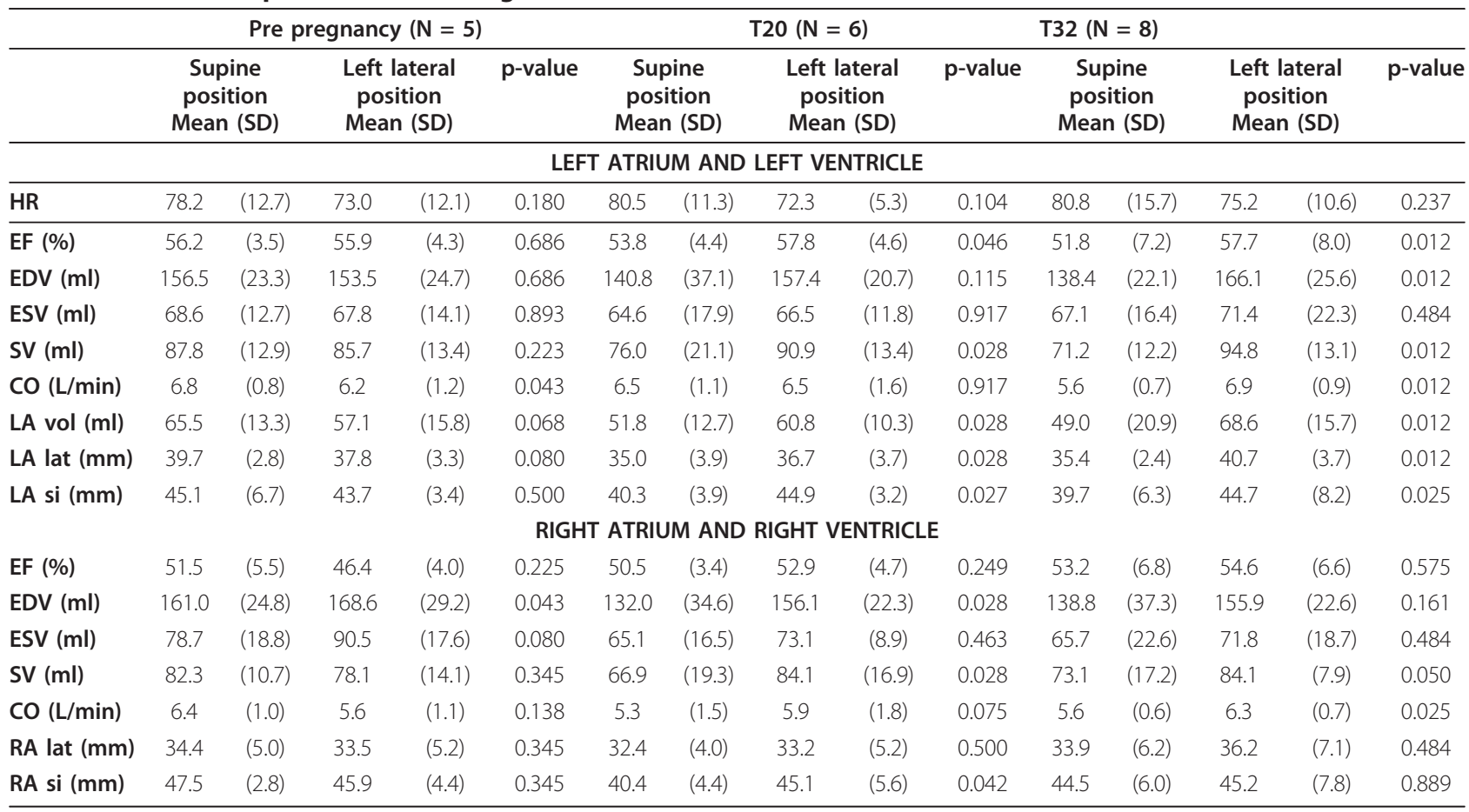

T20: $20^{\text {th }}$ gestational week; T32: $32^{\text {nd }}$ gestational week.

EF (ejection fraction), EDV (end-diastolic volume), ESV (end-systolic volume), SV (stroke volume), CO (cardiac output), LAvol (left atrium volume), LAlat (left atrium lateral diameter), LAsi (left atrium supero-inferior diameter), RAlat (right atrium lateral diameter), RAsi (right atrium supero-inferior diameter)

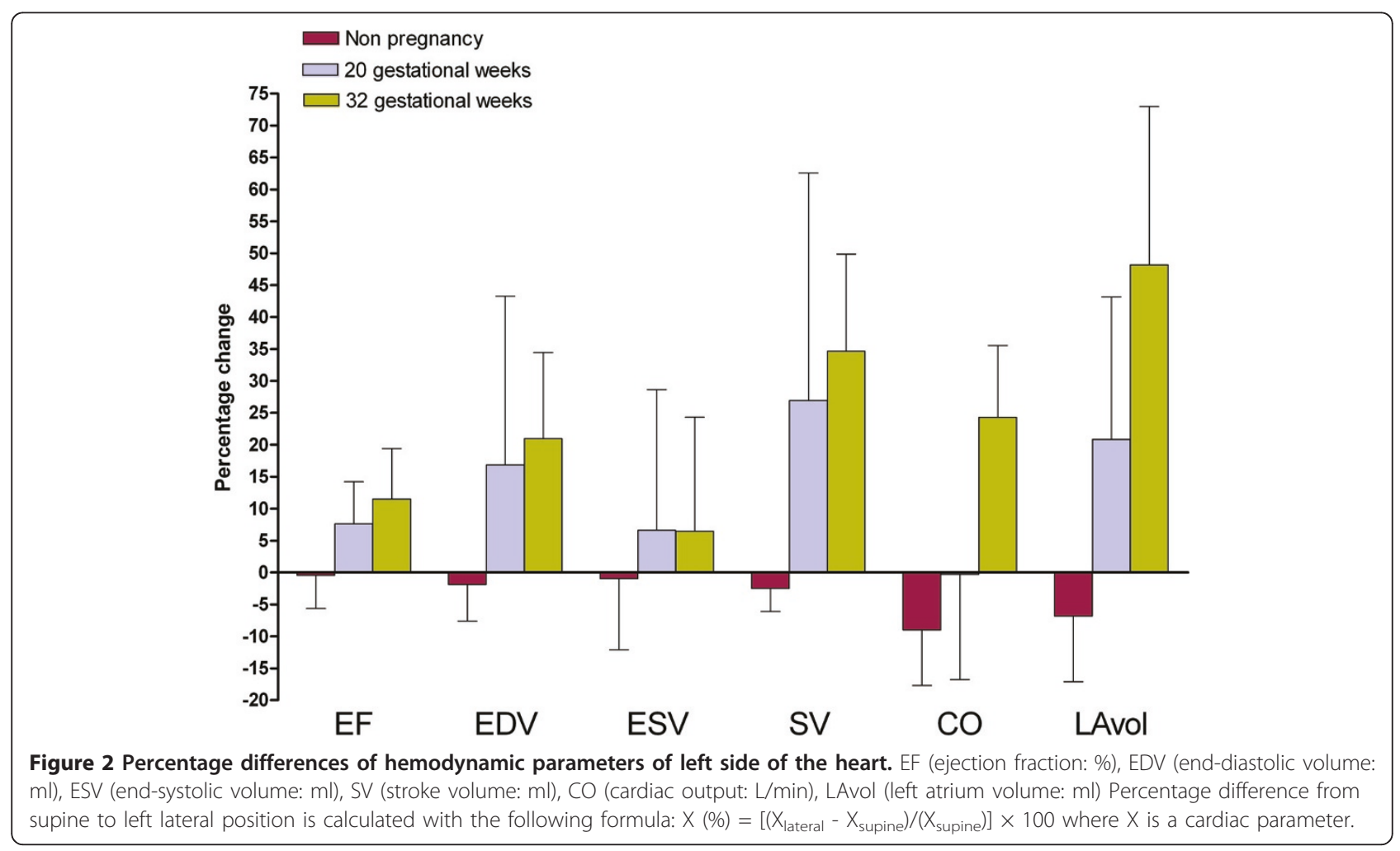




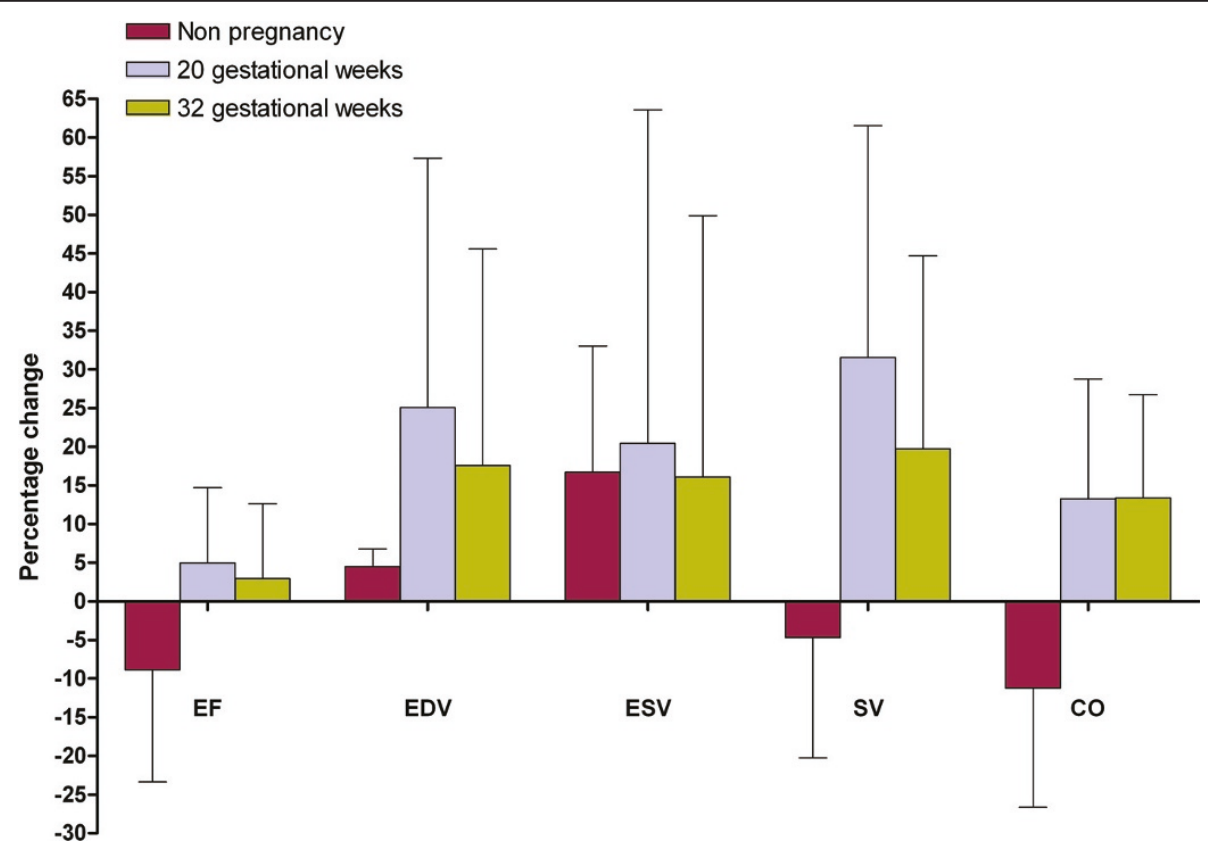

Figure 3 Percentage differences of hemodynamic parameters of right side of the heart. EF (ejection fraction: \%), EDV (end-diastolic volume: $\mathrm{ml}$ ), ESV (end-systolic volume: $\mathrm{ml})$, SV (stroke volume: $\mathrm{ml}$ ), CO (cardiac output: L/min), LAvol (left atrium volume: $\mathrm{ml}$ ) Percentage difference from supine to left lateral position is calculated with the following formula: $X(\%)=\left[\left(X_{\text {lateral }}-X_{\text {supine }}\right) /\left(X_{\text {supine }}\right)\right] \times 100$ where $X$ is a cardiac parameter.

atrial dimensions increased significantly between the supine and the left lateral position by $15 \%$ for LAlat ( $\mathrm{p}$ $=0.012)$ and by $13 \%$ for LAsi $(\mathrm{p}=0.025)$. No significant changes of right hemodynamic parameters and dimensions were observed between the two recumbent positions with the only exception of $\mathrm{CO}(\mathrm{p}=0.025)$.

\section{Impact of gestational age}

A progressive increase of percentage changes of hemodynamic parameters and cardiac dimensions of the left side of the heart was found throughout gestation. The only exception was the percentage change of ESV which substantially did not change between 20 and 32 weeks of gestation. During late pregnancy left ventricle CO significantly increased between supine and left lateral position; the percentage of increment at 32 weeks was $24 \%$. This was associated to an increase of $21 \%$ of left ventricle EDV. A significant increase in LA dimensions was also found. The difference between the two recumbent positions was less clear at $20^{\text {th }}$ gestational week with an increase of left ventricle EDV and LA dimensions but with no clear impact on CO. For the right side of the heart we observed a similar trend of increment of ventricular and atrial dimensions at 20 weeks but no further increase during late pregnancy.

\section{Discussion}

This study investigated how the supine and the left lateral positions during CMR affects heart rate, cardiac volumes and dimensions at different gestational ages. To our knowledge this is the first study investigating the effect of two recumbent positions on cardiovascular hemodynamic measurements and changes in cardiac dimensions during pregnancy using CMR. The data show a clear difference between the two positions, which become more marked as pregnancy advances but are significant from as early as 20 weeks. There were minimal changes in the non-pregnant subjects. The existing data from non-pregnant humans with regards to the effect of different positions on hemodynamic parameters are limited and sometimes conflicting. Some report that cardiac output is higher in the supine position compared to the right or left lateral position $[12,13]$, while others have shown that cardiac output is higher in the left lateral position than in the supine position [14] and still others that there is no difference in either position $[15,16]$. In our series, we found that there was no significant effect on hemodynamic parameters and cardiac dimensions of moving from the supine to the left lateral position in non-pregnant women. However, we did observe a non significant decline in both heart rate and stroke volume leading to a borderline significant reduction in cardiac output.

Pregnancy itself is a circulatory burden with a significant impact on the cardiovascular system. Cardiac output increases 30-50\% above pre-pregnancy levels. In addition, when a pregnant woman lies flat on her back, 
the gravid uterus partially compresses the inferior vena cava with the consequent reduction of venous filling load and cardiac output [2,17]. From our data it appears that the increase of left atrial volume accounts for the majority in increase in stroke volume. As such it seems that the relief of caval obstruction (preload) is far more important that the relief of aortic compression (afterload) for the increase in cardiac output. In our series the significant increase in LA dimensions and the trend to increase EDV in the left lateral position suggest an increased venous return in this position which is already present at 20 gestational weeks. The effect of the gravid uterus compressing the abdominal vessels might be enforced by the increase in plasma volume even as early as 20 weeks of pregnancy [2]. An increase of right atrial pressure [18] and left and right ventricular peak systolic and end-diastolic pressures [19] in the left lateral position can also help to explain the increased venous return. It is of interest to observe that the heart rate was higher in the supine position compensating for the fall in stroke volume in this position [16] in an attempt to recover cardiac output. This could be partly explained by the fact that during mid-late pregnancy the suppression of cardiac vagal activity and the enhancement of cardiac sympathetic activity are greater in the supine position [20] than in the lateral position. In this series left ventricle EDV, EF, CO and SV increase significantly from the supine to left lateral position. These findings are easily explained: the change from the supine to the lateral position relieves the compression on the vena cava from the gravid uterus. The increasing venous return leads to an increased SV and so CO. Ueland et al [16] demonstrated that a change in position from the supine to the left lateral side produced a rise in $\mathrm{CO}$ by $8 \%$ at 20 to 24 weeks gestation, $13.6 \%$ at 28 to 32 weeks gestation and $28.5 \%$ at term in a group of eleven healthy pregnant women. We observed that the SV increased by approximately $27 \%$ at 20 weeks gestation. At 32 weeks gestation SV increased significantly by $35 \%$. In our series turning to the left lateral position we observed an increment of the SV of the right ventricle which is more evident in the mid than in the late pregnancy. More studies are needed to better explain the consistency of this finding.

Some concerns may develop regarding the use of CMR in pregnant patients. Most studies evaluating MR safety during pregnancy do not show ill effects on the fetus [21-23]. It is anyway good practice to avoid MR studies during the first trimester of pregnancy although it can be used if clinical indicated. All our patients were studied during the second or third trimester of pregnancy.

Several limitations of this study should be highlighted. First, our results should be tested in a larger sample of women including women with cardiac disease. Indeed the normal physiological respond to pregnancy could be different in patients with congenital or acquired cardiovascular diseases. In addition, the small sample size may justify the large standard deviation in our series. Second, because the interval between each MR acquisition was between 8 and 12 minutes it is possible that cardiac parameters had not returned to baseline. Additional studies using more time points and also investigating the reverse change from lateral to supine could clarify this interesting subject. Third, this study is a cross-sectional study. The effects of the position on cardiac hemodynamic should preferably be studied in a prospectivelongitudinal study investigating the same population at different gestational times.

\section{Conclusion}

In the non-pregnant state, turning from supine to left lateral position has minimal effect on cardiac parameters. During pregnancy, from as early as 20 weeks, turning to the left lateral position has positive effects on cardiac hemodynamics inducing a significant increase of venous return, SV and CO. Pregnant women requiring CMR should be studied in a consistent position for serial studies and the left lateral position is preferred from early pregnancy onwards, also to limit uteroplacental hypoperfusion.

\section{List of abbreviations}

CMR: cardiovascular magnetic resonance; SA: short-axis; EDV: end-diastolic volume; ESV: end-systolic volume; EF: ejection fraction; SV: stroke volume; CO: cardiac output; LV: left ventricle; RV: right ventricle; LA: left atrium; RA: right atrium; LAlat: lateral left atrium diameter; LAsi: supero-inferior left atrium diameter; RAlat: lateral right atrium diameter; RAsi: supero-inferior right atrium diameter

\section{Author details}

${ }^{1}$ Department of Cardiology, Erasmus University Medical Center, 's Gravendijkwal 230, 3015CE Rotterdam, The Netherlands. 'Department of Radiology, Erasmus University Medical Center, 's Gravendijkwal 230, 3015CE Rotterdam, The Netherlands. 'Department of Obstetrics and Gynaecology, Division of Obstetrics and Prenatal Medicine, Erasmus University Medical Center, 's Gravendijkwal 230, 3015CE Rotterdam, the Netherlands. ${ }^{4}$ Department of Obstetrics and Gynaecology, Imperial College of Medicine, Chelsea and Westminster Hospital, London, UK.

\section{Authors' contributions}

The contributions of each author to this manuscript are as follows; AR was involved in the acquisition, analysis and interpretation of the data and drafting of the manuscript. YK, TS and PO were involved in the conception and acquisition of the data. JC, MRJ, AM, GPK, ES were involved in revising the manuscript critically for important intellectual content. JRH was involved in the design of the study and revising the manuscript. RJG was responsible for the conception and design of the study, interpretation of the data and drafting the manuscript. All authors have read and approved the final manuscript.

\section{Competing interests}

The authors declare that they have no competing interests.

Received: 17 January 2011 Accepted: 27 June 2011

Published: 27 June 2011 


\section{References}

1. Drenthen W, Pieper PG, Roos-Hesselink JW, van Lottum WA, Voors AA Mulder BJ, van Dijk AP, Vliegen HW, Yap SC, Moons P, et al: Outcome of pregnancy in women with congenital heart disease: a literature review. J Am Coll Cardiol 2007, 49:2303-2311.

2. Karamermer $Y$, Roos-Hesselink JW: Pregnancy and adult congenital heart disease. Expert Rev Cardiovasc Ther 2007, 5:859-869.

3. Uebing A, Arvanitis P, Li W, Diller GP, Babu-Narayan SV, Okonko D Koltsida E, Papadopoulos M, Johnson MR, Lupton MG, et al: Effect of pregnancy on clinical status and ventricular function in women with heart disease. Int J Cardiol 2010, 139:50-59.

4. Tzemos N, Silversides CK, Colman JM, Therrien J, Webb GD, Mason J, Cocoara E, Sermer M, Siu SC: Late cardiac outcomes after pregnancy in women with congenital aortic stenosis. Am Heart J 2009, 157:474-480.

5. Kilner PJ, Geva T, Kaemmerer H, Trindade PT, Schwitter J, Webb GD: Recommendations for cardiovascular magnetic resonance in adults with congenital heart disease from the respective working groups of the European Society of Cardiology. Eur Heart J 2010, 31:794-805.

6. Del Bene R, Barletta G, Mello G, Lazzeri C, Mecacci F, Parretti E, Martini E, Vecchiarino S, Franchi F, La Villa G: Cardiovascular function in pregnancy: effects of posture. BJOG 2001, 108:344-352.

7. Flo K, Wilsgaard T, Vartun A, Acharya G: A longitudinal study of the relationship between maternal cardiac output measured by impedance cardiography and uterine artery blood flow in the second half of pregnancy. BJOG 2010, 117:837-844.

8. Pump B, Talleruphuus U, Christensen NJ, Warberg J, Norsk P: Effects of supine, prone, and lateral positions on cardiovascular and renal variables in humans. Am J Physiol Regul Integr Comp Physiol 2002, 283: R174-180

9. Kirschbaum SW, Baks T, Gronenschild EH, Aben JP, Weustink AC, Wielopolski PA, Krestin GP, de Feyter PJ, van Geuns RJ: Addition of the long-axis information to short-axis contours reduces interstudy variability of left-ventricular analysis in cardiac magnetic resonance studies. Invest Radiol 2008, 43:1-6.

10. Sievers B, Kirchberg S, Bakan A, Franken U, Trappe HJ: Impact of papillary muscles in ventricular volume and ejection fraction assessment by cardiovascular magnetic resonance. J Cardiovasc Magn Reson 2004, 6:9-16.

11. van Geuns RJ, Baks T, Gronenschild EH, Aben JP, Wielopolski PA, Cademartiri F, de Feyter PJ: Automatic quantitative left ventricular analysis of cine MR images by using three-dimensional information for contour detection. Radiology 2006, 240:215-221.

12. Newman B, Derrington C, Dore C: Cardiac output and the recumbent position in late pregnancy. Anaesthesia 1983, 38:332-335.

13. Atkins AJ, Watt JM, Milan P, Davies P, Crawford JS: The influence of posture upon cardiovascular dynamics throughout pregnancy. Eur J Obstet Gynecol Reprod Biol 1981, 12:357-372.

14. Doering L, Dracup K: Comparisons of cardiac output in supine and lateral positions. Nurs Res 1988, 37:114-118.

15. Lange RA, Katz J, McBride W, Moore DM Jr, Hillis LD: Effects of supine and lateral positions on cardiac output and intracardiac pressures. Am $J$ Cardiol 1988, 62:330-333.

16. Ueland K, Novy MJ, Peterson EN, Metcalfe J: Maternal cardiovascular dynamics. IV. The influence of gestational age on the maternal cardiovascular response to posture and exercise. Am J Obstet Gynecol 1969, 104:856-864

17. Robson SC, Hunter S, Boys RJ, Dunlop W: Serial study of factors influencing changes in cardiac output during human pregnancy. Am J Physiol 1989, 256:H1060-1065.

18. Lees MM, Scott DB, Kerr MG, Taylor SH: The circulatory effects of recumbent postural change in late pregnancy. Clin Sci 1967, 32:453-465.

19. Nakao S, Come PC, Miller MJ, Momomura S, Sahagian P, Ransil BJ, Grossman W: Effects of supine and lateral positions on cardiac output and intracardiac pressures: an experimental study. Circulation 1986, 73:579-585.

20. Kuo CD, Chen GY, Yang MJ, Tsai YS: The effect of position on autonomic nervous activity in late pregnancy. Anaesthesia 1997, 52:1161-1165.

21. Clements H, Stephenson T, Gabriel V, Harrison T, Millar M, Smyth A, Tong W, Linton CJ: Rationalised prescribing for community acquired pneumonia: a closed loop audit. Arch Dis Child 2000, 83:320-324.

22. Kok RD, de Vries MM, Heerschap A, van den Berg PP: Absence of harmful effects of magnetic resonance exposure at $1.5 \mathrm{~T}$ in utero during the third trimester of pregnancy: a follow-up study. Magn Reson Imaging 2004, 22:851-854.

23. Kanal E, Barkovich AJ, Bell C, Borgstede JP, Bradley WG Jr, Froelich JW, Gilk T, Gimbel JR, Gosbee J, Kuhni-Kaminski E, et al: ACR guidance document for safe MR practices: 2007. AJR Am J Roentgenol 2007, 188:1447-1474

doi:10.1186/1532-429X-13-31

Cite this article as: Rossi et al:: Quantitative cardiovascular magnetic resonance in pregnant women: cross-sectional analysis of physiological parameters throughout pregnancy and the impact of the supine position. Journal of Cardiovascular Magnetic Resonance 2011 13:31.

\section{Submit your next manuscript to BioMed Central and take full advantage of:}

- Convenient online submission

- Thorough peer review

- No space constraints or color figure charges

- Immediate publication on acceptance

- Inclusion in PubMed, CAS, Scopus and Google Scholar

- Research which is freely available for redistribution

Submit your manuscript at www.biomedcentral.com/submit
C Biomed Central 